\title{
A NOVEL METHOD FOR ELECTROMAGNETIC DOSIMETRY RELATED TO HUMAN EXPOSURE FROM SHORT-RANGE DEVICES
}

\author{
D. ŠIMUNIĆ \& D. ZRNO \\ Department for Radiocommunications, Faculty of Electrical Engineering and \\ Computing, University of Zagreb, Zagreb, Croatia.
}

\begin{abstract}
Electromagnetic dosimetry, which quantifies the interaction of electromagnetic fields with biological material, is primarily used for the evaluation of human exposures due to mobile wireless devices generating electromagnetic fields. The term dosimetry refers to the absorbed energy in an object, while exposimetry encompasses external field measurements. Both dosimetry and exposimetry are based on experimental and/or numerical methods. In this paper a proposed new method for numerical exposimetry for short-range radio frequency devices is described. The novelty of the field calculation method, called beam-tracing, lies in the determination of the total electromagnetic field, i.e. direct, reflected, transmitted, and diffracted, at a specific location. The calculation of the total external electromagnetic fields enables the determination of the deposited electromagnetic energy in a human being through the specific absorption rate using known approximation formulas. This paper presents an application of the beam-tracing method to calculations of human exposure from typical wireless local-area network systems located in an indoor environment.
\end{abstract}

Keywords: beam-tracing, exposimetry, non-ionizing radiation dosimetry, SAR, short-range devices.

\section{INTRODUCTION}

Electromagnetic (EM) dosimetry is defined in [1] as the evaluation of energy absorbed by an object exposed to radio frequency (RF) EM fields. In EM dosimetry, the absorbed energy is determined by the internal EM fields that are induced by external (incident) EM energy. The external and internal fields are very different and are interrelated by the EM field boundary conditions and wave transmission theory. The important parameters are the size and shape of the biological object (human body), its electrical properties, its orientation with respect to the incident EM fields, and the frequency of the incident fields. The reasoning that direct determination is much easier for external rather than internal EM fields in an object/body gives exposimetry an important advantage [2]. Once the exposimetry results are known, it is advisable to apply dosimetry for relating the internal to external fields. This relation depends on the shape of the object, its composition, and material properties, as well as on the characteristics of the incident EM field.

Short-range devices (SRDs) are radio devices that exhibit a low potential for interference to other radio services, usually because their transmitted power and, hence, their range, is relatively small. SRDs often operate in privileged conditions with respect to radio regulations, meaning users do not need individual licenses to operate such equipment. Other information about SRD issues is given in Section 2 below. Section 3 provides general explanations about the biological effects of EM fields.

The term 'beam-tracing' has typically been applied to two different approaches for field calculations. The first approach is a ray-shooting algorithm [3] in which many rays aggregate to form a beam. The second approach relies on a method of images [4]. The methods used in this paper are based on the second approach. Direct, reflected, transmitted, and diffracted EM field strength values were calculated at specific indoor locations in buildings, in the absence of a human body. The representation of a typical indoor environment is simplified by modeling only objects which contribute most significantly to the wave propagation. The most important property of the method described is that physically non-feasible reflection combinations, i.e. higher order reflections, transmissions, and

() 2006 WIT Press, www.witpress.com

ISSN: 1743-5242 (paper format), ISSN: 1743-5250 (online), http://journals.witpress.com DOI: $10.2495 / \mathrm{ECO}-\mathrm{V} 1-\mathrm{N} 4-327-338$ 
diffractions, are eliminated. The specific beam-tracing implementation presented was developed at the Faculty of Electrical Engineering and Computing at the University of Zagreb, and is described in Section 4.

Calculations have been performed for indoor locations served by signals from a wireless local-area network (WLAN) access point (AP) in the $2.4 \mathrm{GHz}$ band. Calculated EM fields were verified using measurements of the same system. The measurements also helped in fine-tuning the environmental parameters used in the calculations. The measurement method and results are presented in Section 5.

The comparison between computed and measured results is given in Section 6. The main points about dosimetry and conclusions about the final dosimetric results are given in Section 7, followed by final conclusions in Section 8 .

\section{SHORT-RANGE DEVICES}

In the European regulatory domain, the European conference of Posts and Telecommunications (CEPT) defines 13 different applications of SRDs [5] as follows, with corresponding maximum radiated power or EM field strength limits: non-specific SRD (telemetry, telecommand, alarms, speech, and video), devices for detecting avalanche victims, WLANs, automatic vehicle identification for railways, road transport, and traffic telematics, equipment for detecting movement and for alert, alarms, and model control, inductive applications, radio microphones, RF identification systems, ultra-low-power active medical implants, and wireless audio applications.

A SRD uses either an integral or an external antenna, with all relevant standard modes of modulation. In Europe, SRDs have to meet the Radio and Telecommunications Terminal Equipment (R\&TTE) Directive [6] to permit placing on the market within the European Community. The R\&TTE that can be placed on the market and be put into service without restrictions are known as 'Class 1' equipment [7]. The type of SRD considered in this paper is WLAN equipment, which operates in the unlicensed $2.4 \mathrm{GHz}$ band as Class 1 equipment (according to 2000/299/EC). The RF radiated power limit for WLAN devices is $100 \mathrm{~mW}$ EIRP. For systems that use direct-sequence spread-spectrum modulation, the maximum spectral power density is limited to $-20 \mathrm{dBW} /(1 \mathrm{MHz})$. For equipment that uses frequency-hopping spread-spectrum modulation, the maximum spectral power density is limited to $-10 \mathrm{dBW} /(100 \mathrm{kHz})$.

\section{BIOLOGICAL EFFECTS OF EM FIELDS}

The present state-of-the-art conclusions about any possible biological effects of EM fields maintain that even high-intensity RF EM fields cannot cause ionization in biological systems. The effects confirmed by scientists include heating and the induction of electrical currents in tissues. Even very low levels of energy produce a small amount of heat by moving ions and water molecules throughout the medium in which they exist, but this heat is dissipated by normal thermoregulatory processes and is thus indiscernible from normal human activities. The results of some studies suggest that exposure to fields which are too weak to cause heating may have adverse health consequences, including cancer and memory loss, but these findings have not been confirmed by the overall bioelectromagnetics research community [8].

\section{EM FIELD CALCULATIONS}

Calculations of the EM fields generated by a typical WLAN system at certain nearby positions have been performed using the beam-tracing calculation software [9] developed by our group at the University of Zagreb. In general, EM wave propagation is a complex phenomenon, and this complexity increases in the case of propagation in indoor environments. Indoor environments typically contain many objects that can influence propagation, meaning that EM waves experience direct-ray 
propagation as well as the phenomena of reflection, diffraction, and transmission affected by all the objects present. These effects create multi-path propagation conditions, characterized by a possible direct ray between the transmitter and receiver, and a large number of lower amplitude rays reaching any observation point after multiple reflections or diffractions. Errors in average power estimations with and without accounting for small-scale fading estimated for a typical GSM system [10] have shown that these effects are not negligible. Therefore, a balance between the calculation resources and accuracy is needed. Some published results based on the ray-tracing method [11] consider only direct and reflected EM fields. Besides direct and reflected fields, the method used here also takes into account transmitted and diffracted fields in the absence of a human body. However, our method neglects reflection combinations that are not physically feasible, i.e. higher order reflections, transmissions, and diffractions. In general, indoor environments consist of many objects. For the sake of computational resources, indoor environments are simplified here by modeling only those objects that most significantly influence propagation. On the other hand, care has been taken not to oversimplify the representation of the environment to avoid larger errors.

For inputs, the software developed requires three data types as described below: an environment map, transceiver equipment parameters, and propagation parameters.

The 'environment map' is a file containing the spatial coordinates for all objects in the environment, and the object type, such as walls, doors, and windows along with their electrical properties. These parameters are defined by the user in a map-editing utility program which was developed specifically for this purpose. Reflection and transmission losses are calculated from the input material and environmental properties.

'Equipment parameters' required for the calculation include only the most relevant information for the transmitter and receiver equipment used. Transmitter parameters include carrier frequency ( $f$, from which the signal wavelength $\lambda$ is calculated), transmitter power $\left(P_{\mathrm{Tx}}\right)$, and transmitter antenna parameters, i.e. antenna gain $\left(G_{\mathrm{Tx}}\right)$, radiation pattern, and antenna orientation. Receiver parameters include received power $\left(P_{\mathrm{Rx}}\right)$, receiver antenna gain $\left(G_{\mathrm{Rx}}\right)$, receiver noise figure, bandwidth of the received signal, and receiver temperature. These are used to calculate receiver sensitivity, which determines minimum receivable signal strength.

'Propagation parameters' include definition of the fading margin and the propagation exponent. The fading margin is an overhead in signal strength required to counter the effects of sudden drops in received signal power (called signal fading) caused by changes in the environment. The fading margin required depends on the desired connection quality and is usually set between 10 and $20 \mathrm{~dB}$ for typical indoor environments. The propagation exponent $[R$, as used in eqn (1)] determines the falloff of signal power density with distance from the transmitter. When the propagation exponent equals 2 , the equation becomes the classic free-space transmission formula [12]. For typical indoor areas, many small objects existing in an office environment contribute to the EM wave reflection, diffraction, and scattering, such that the free-space propagation exponent is no longer applicable. Typical indoor environments entail modification of the free-space propagation exponent to a value between 2 and 6.5 depending on the number of the obstacles present [13]. In this work, the environment map considered contains a number of relatively large objects, e.g. walls, but smaller objects have been neglected. Therefore, a novelty of the model here is that it uses a modification of the propagation exponent in the Friis' free-space transmission formula. The value is not 2 as is used for free space, but a value of the propagation exponent $R$ to calculate the received signal power at a distance $d$ from the transmitter for each ray, indexed as $j$ and going from 1 to $m$, that reaches the receiver, as given in eqn (1):

$$
P_{\mathrm{Rx}}=\sum_{j=1}^{m}\left(\frac{P_{\mathrm{Tx}} G_{\mathrm{Tx}} G_{\mathrm{Rx}}}{\prod_{i=1}^{n} L_{\mathrm{d} i} \cdot L_{\mathrm{f} i} \cdot L_{\mathrm{c} i}}\left(\frac{\lambda}{4 \pi}\right)^{2} \frac{1}{d^{R}}\right)_{j} .
$$


Influences on received signal strength from the 'ith' obstacle (counted from 1 to $n$ ) within the environment are accounted for by the attenuation propagation parameters of each obstacle, i.e. its diffraction loss, $L_{\mathrm{d}}$, signal loss due to fading, $L_{f}$, and cable and connector losses, $L_{\mathrm{c}}$.

The propagation exponent chosen is 'tuned' by comparison with measurement results, using a test set-up and the methods described below. The most appropriate 'fit' value for the environments considered here was found to be 2.3 .

The software models the basic propagation phenomena of transmission, reflection, and diffraction using the Kirchoff-Huygens principle to predict signal strengths by application of Fresnel's equations [14]. The accuracy of calculation depends upon the accuracy of the input objects' electrical properties, as well as upon beam density. Another novelty of the method, compared to work by other authors $[15,16]$, is that it bundles rays subject to the same propagation phenomena into beams. In this 'beamtracing' method, each beam is represented by a signal image, which is a matrix containing signal strength values within a given beam area. The beam area is a two-dimensional cross-section of a beam tube in a plane perpendicular to the propagation direction. The calculations apply geometrical-optics transformations to the entire beam area, thereby accelerating the calculation process considerably. Unlike the ray-tracing method, which calculates signal strength for each map point separately, the beam-tracing method calculates signal strengths for the entire map simultaneously.

The beam-tracing algorithm utilizes signal images that cover an area larger than the observed environment. The initial signal image in matrix form is created for an obstacle-free environment using the modified free-space equation [eqn (I)]. This matrix is fed into a recursive function, which is called repeatedly each time after spawning a new matrix. The algorithm searches the input environment database for objects found in a given matrix area having sufficient local signal strength for reception. The software determines which beam encountered the object and separates this beam to make a different signal image, which is fed back into the recursive function. This process is done separately for each propagation effect, producing three new image matrices that are combined and used to modify the original signal image. In this way, a tree of all possible propagation paths throughout the entire environment is created. The main advantage of this approach, compared to standard ray-tracing methods, is that this model retains its spatial resolution independent of the distance between receiver and transmitter.

The calculation process begins with an environment parameters window to input the spatial coordinates and the electrical properties of objects within the environment. The next step in the calculation uses the graphical interface shown in Fig. 1. This screen is used for input of propagation parameters and for presentation of the results. Input fields for equipment and propagation parameters are on the right side of the interface, grouped into three boxes; output files can be defined for calculations performed with and without diffraction; calculation results are shown on the bottom part of the display. One of the results is a display of the power level with and without diffraction at a local position indicated by the cursor position on the environment map; the other is a percentage of the defined area that has sufficient signal strength for reception (i.e. above the sensitivity level increased by the fading margin). Areas with signal levels for unsuitable-quality communications link are marked white; the remaining areas are scaled from light gray (just sufficient to establish a communications link) to black (maximum signal quality). The shaded bar next to the signal image enables the user to make a rough estimation of the signal strength, expressed as received power level, in areas where communications are possible. More accurate readings are obtained by simply placing the cursor over any map point, because one display field always shows the pointer position and the signal strength for the cursor location. If needed, for a more detailed study of the propagation, the software also enables the three-dimensional visualization of the environment and of the calculation results, as well as a comparison between calculations with and without diffraction, or between calculations using varied input parameters. 


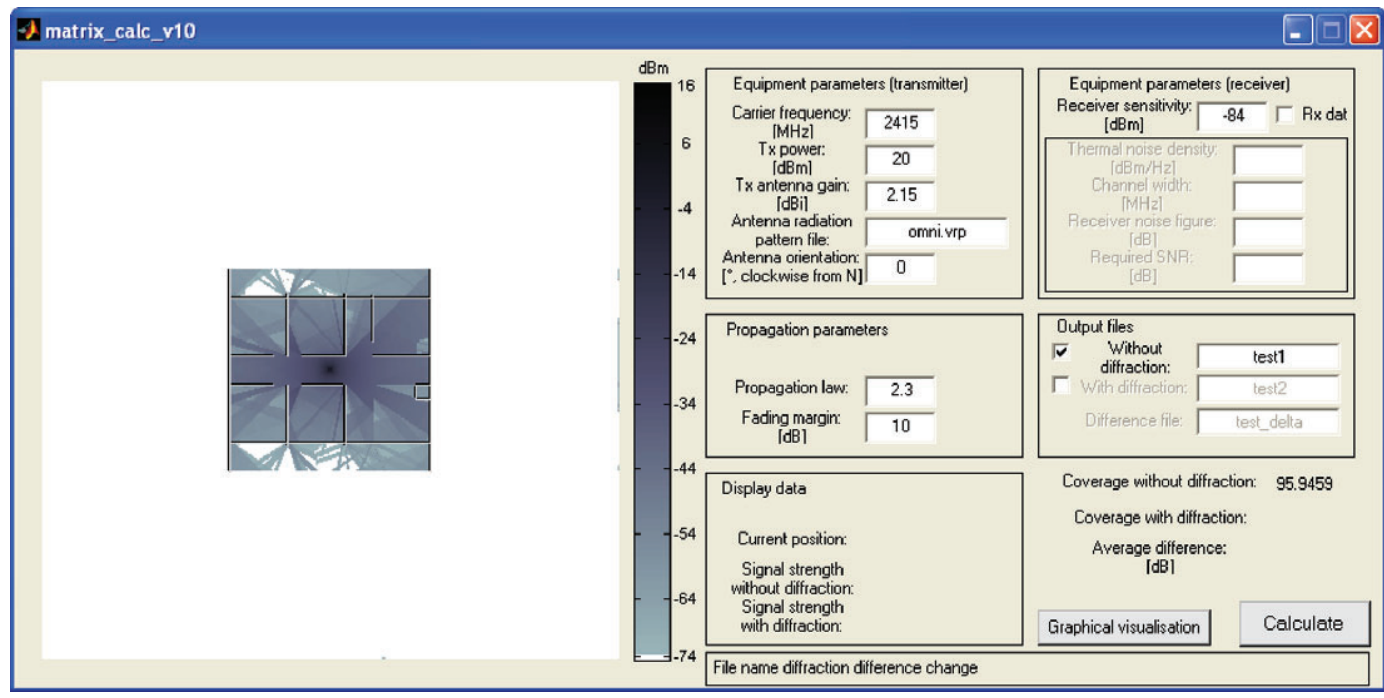

Figure 1: Graphical user interface of the beam-tracing software package. Descriptions of display items and parameters for the example shown are given in the text.

The example in Fig. 1 shows equipment parameters (transmitter and receiver) inputs for a WLAN system operating on the first channel, with $20 \mathrm{dBm}$ transmitter power and 2.15 transmitter antenna gain. The receiver sensitivity is $-84 \mathrm{dBm}$. The input propagation parameters are: a propagation law exponent of 2.3 as well as a fading margin of $10 \mathrm{~dB}$. In this example, the output file is calculated without diffraction. The bottom part of the window shows the present cursor position with the corresponding value of the signal strength without diffraction. The 'Coverage' results express the area of received signal strength sufficient to have successful communications, reported as a percentage of the entire environment map area. The 'Graphical visualisation' button allows the viewing of calculation results with and without diffraction. The graphical display in the left half of the screen shows the distribution of signal strengths in the defined space, where the black color indicates the source location (WLAN AP, in this example) with the highest signal strength and light gray indicates areas with lower power signal strengths. The white color shows areas with insufficient signal strengths to support successful communication.

\section{MEASUREMENT METHOD}

The validation and fine-tuning of the calculation method was done using comparisons to a series of measurement results. A WLAN (802.11b protocol [17]) was used in the measurements as a typical system for which the beam-tracing software can be applied. The limited transmitter range for typical WLANs allows measurements over the entire coverage area with relatively high resolution. Measurements were done using a single WLAN AP operating on the fifth channel at $2.432 \mathrm{GHz}$ with output power of $18 \mathrm{dBm}$. Two dipole antennas were used for transmission (diversity), and the AP was placed on a foamed polystyrene platform at a height of $1.5 \mathrm{~m}$.

The client device was a laptop computer with a wireless integral-antenna PCMCIA card. The laptop was positioned at a height of $0.9 \mathrm{~m}$ and placed on a wheeled cart for easier transportation and to maintain constant receiver height while performing mobile measurements. Actual network operating conditions were simulated using a software package obtained from the AP manufacturer, 
and measurements at each observation point were performed over a period of 10-20 s. The results were stored and processed using self-written utility software. During the measurements, fading effects were seen as $10-30 \mathrm{~dB}$ decreases in signal strengths, but these effects were reduced by averaging of readings at each measurement point.

\section{COMPARISONS OF CALCULATED AND MEASURED RESULTS}

A comparison between calculated and measured results was performed using an environment map of one floor in a twelve-story building, which houses the Department for Radiocommunications, Faculty of Electrical Engineering and Computing at the University of Zagreb, Croatia. The transmission and reflection loss values used for objects included in the map are given in Table 1.

The environment consisted mainly of concrete walls, wooden doors, and large glass windows on the outside walls, including a total of thirteen offices, four large classrooms, one kitchen, two lavatories, one entry hallway, one main corridor, and four elevators. The entire area was $15 \mathrm{~m}$ wide and $52 \mathrm{~m}$ long and the resolution used for calculations was $0.25 \mathrm{~m}$. Field measurements were made at a number of key locations and along several routes to accumulate sufficient amount of data for model validation. Other calculation parameters were as follows: $P_{\mathrm{Tx}}=18 \mathrm{dBm}, G_{\mathrm{Tx}}=2 \mathrm{dBi}$, $f=2.432 \mathrm{GHz}$, and $R=2.3$. The corresponding beam-tracing calculation results are shown in Fig. 2. The source was located in the room at the upper right-hand side of the figure, where the

Table 1: Values of transmission and reflection losses in $\mathrm{dB}$ used in the calculation example.

\begin{tabular}{lcc}
\hline Type of environment & Transmission loss & Reflection loss \\
\hline Concrete wall & 16 & 6 \\
Window & 6 & 15 \\
Door & 3 & 20 \\
Elevator door & 80 & 1 \\
\hline
\end{tabular}

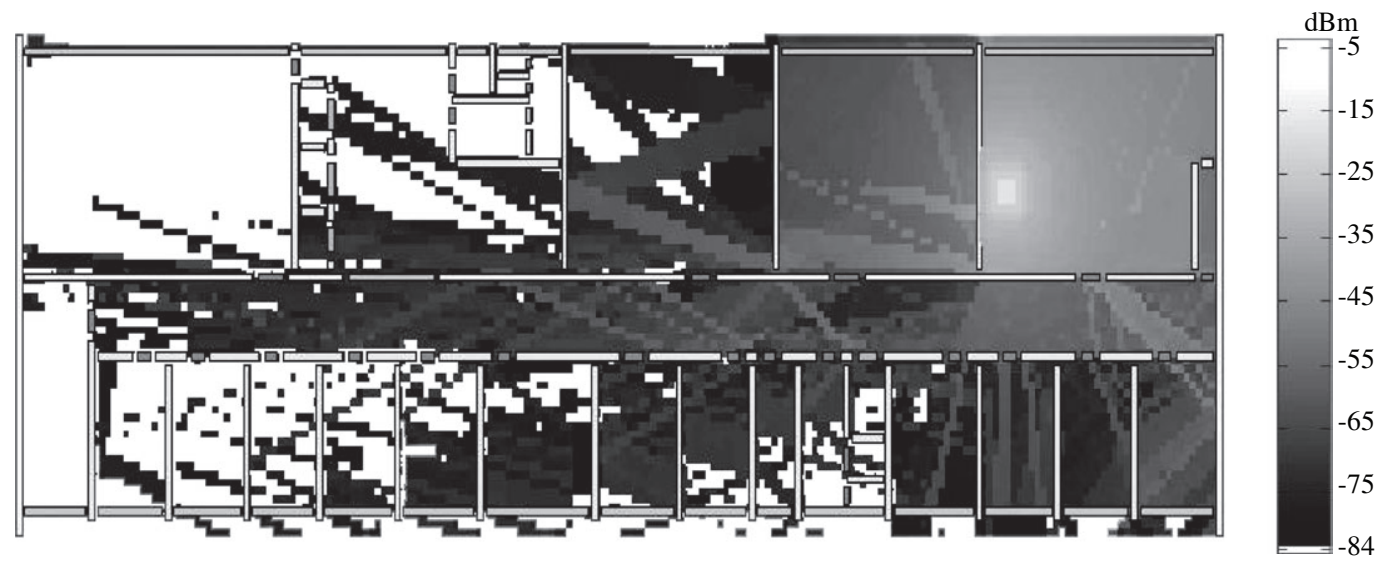

Figure 2: Example beam-tracing calculation results showing distribution of the received signal strength in a typical single floor of a multi-story office building. 


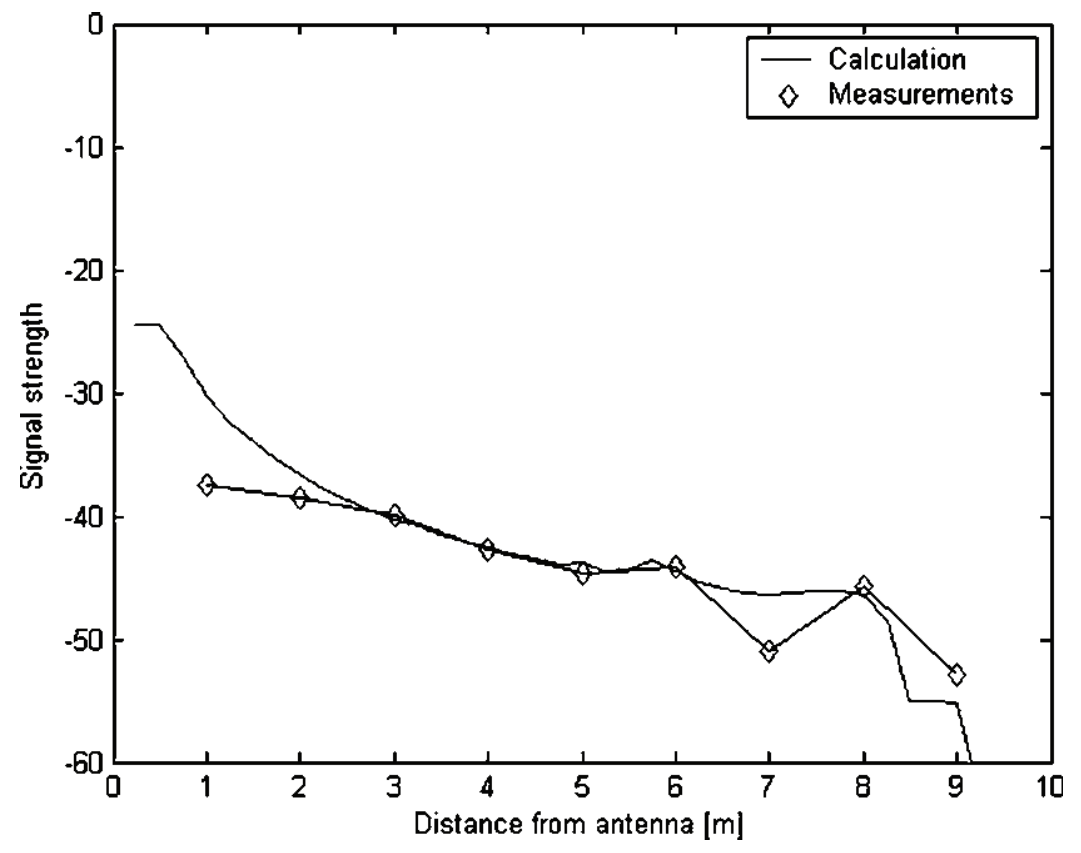

Figure 3: Comparison of measurements and calculation within the room containing the AP.

highest received power level was found. Diffraction and reflection effects were the most pronounced in the corridor. The completely white areas, far from the source, are regions with insufficient signal strength reception, for the given receiver sensitivity of $-84 \mathrm{dBm}$, and not including a fading margin.

Figure 3 shows an example comparison of measured and calculated results for the topography of Fig. 2. It is noted that even the perimeter regions, where the measured signal strengths become too weak to support a communications link, show good agreement between calculated and measured results. The model was 'calibrated' using measurement results in the large room where the WLAN AP was placed, as shown in Fig. 2. Measurements were done at points spaced every $1 \mathrm{~m}$ from the $\mathrm{AP}$, and the results were used to derive the model input parameters. The standard deviation between measured points and the corresponding calculated points is $4.2 \mathrm{~dB}$. Thus, from Fig. 3 we can conclude that the measurement results confirm the calculated results fairly well, especially at greater distances from the antenna.

The model parameters were selected to fit the measurement results within the room containing the AP as best as possible. In the next phase, additional measurements were performed in the main corridor. The standard deviation between measured points and corresponding calculated points in the corridor shows a reasonably good correlation of $3.5 \mathrm{~dB}$, which is a very good result for a non-lineof-sight case of EM propagation.

\section{DOSIMETRY RELATED TO HUMAN EXPOSURE}

EM dosimetry is a methodology used for determining compliance with specific absorption rate (SAR) limits applicable for portable and mobile wireless devices intended to be used with the radiating part of the device within $20 \mathrm{~cm}$ of a human body. A convenient analytical approximation formula, as proposed in [18], for calculation of the SAR induced at the surface of the standing human, $S A R_{\text {surf }}$, 
for the case of a monopole antenna with root mean squared (RMS) radiated power $P_{\mathrm{RMS}}$ and feed point impedance $Z$ is as follows:

$$
S A R_{\text {surf }}=\frac{\sigma}{\rho} \frac{\mu \omega}{\sqrt{\sigma^{2}+\varepsilon^{2} \omega^{2}}} \cdot\left(1+c_{\text {corr }} \Gamma\right)^{2}\left[\frac{P_{\mathrm{RMS}}}{4 \pi^{2} d^{2} \operatorname{Re}(Z)}\right] .
$$

The electrical properties for a homogeneous biological tissue model are described by $\sigma$ (conductivity), $\varepsilon$ (permittivity), $\mu$ (permeability), and $\rho$ (tissue density). The parameter $\Gamma$ is the plane-wave reflection coefficient for the incident magnetic field, and $c_{\text {corr }}$ is a correction factor that accounts for the reflection properties for small distances $d$ from the dielectric surface. The frequency considered was $2,450 \mathrm{MHz}$, which means for the considered case $c_{\text {corr }}$ becomes 1 . The quotient expression in the brackets is the square of the incident magnetic field $H_{\text {inc }}$, with $Z_{0}$ being the free-space wave impedance. In other words:

$$
H_{\text {inc }}^{2}=\frac{P_{\mathrm{RMS}}}{4 \pi^{2} d^{2} \operatorname{Re}(Z)}=\frac{E_{\mathrm{inc}}^{2}}{Z_{0}^{2}} .
$$

The reflection coefficient $\Gamma$ is defined in terms of the complex permittivity $\varepsilon^{\prime}$ :

$$
\Gamma=\frac{2\left|\sqrt{\varepsilon^{\prime}}\right|}{\left|\sqrt{\varepsilon^{\prime}}+\sqrt{\varepsilon_{0}}\right|}-1,
$$

where complex permittivity $\varepsilon^{\prime}$ is:

$$
\varepsilon^{\prime}=\varepsilon-j \frac{\sigma}{\omega} .
$$

Rewriting eqn (2) using eqns (3)-(5) in the plane-wave region, the following equation is obtained for $\mathrm{SAR}$ at the body/object surface:

$$
S A R_{\text {surf }}=\frac{\sigma}{\rho} \frac{\mu \omega}{\sqrt{\sigma^{2}+\varepsilon^{2} \omega^{2}}} \cdot\left(\frac{2\left|\sqrt{\varepsilon^{\prime}}\right|}{\left|\sqrt{\varepsilon^{\prime}}+\sqrt{\varepsilon_{0}}\right|}\right)^{2}\left[\frac{E_{\mathrm{inc}}}{Z_{0}}\right]^{2} .
$$

As an example, electrical parameters used in the $2.45 \mathrm{MHz}$ frequency band for the homogeneous all-muscle model are $\varepsilon_{\mathrm{r}}=53.57, \sigma=1.81 \mathrm{~S} / \mathrm{m}$, and $\rho=1040 \mathrm{~kg} / \mathrm{m}^{3}$. The values for an anatomically correct heterogeneous body model have been calculated at a specific frequency on a Federal Communications Commission internet site [19] from the results of Gabriel [20] using the 4-Cole-Cole analysis.

The SAR averaged over a cube of side length $\Delta x, S A R_{\Delta x}$, as used in several human exposure regulations, can be approximated using $S A R_{\text {surf }}$ and eqn (6) [21], for a homogeneous representation of the body with tissue skin depth $\delta$ given by eqn (7), as from [22]:

$$
S A R_{\Delta x} \approx \frac{\delta}{2 \Delta x} S A R_{\text {surf }}\left(1-\mathrm{e}^{-2 \Delta x / \delta}\right) .
$$

The skin depth $\delta$ for a plane wave incident on a planar dielectric with losses [1] is defined as:

$$
\delta=\frac{67.52}{f} \frac{1}{\sqrt{\left[\sqrt{\varepsilon^{2}+\left(\frac{\sigma}{\omega}\right)^{2}}-\varepsilon\right]}} .
$$




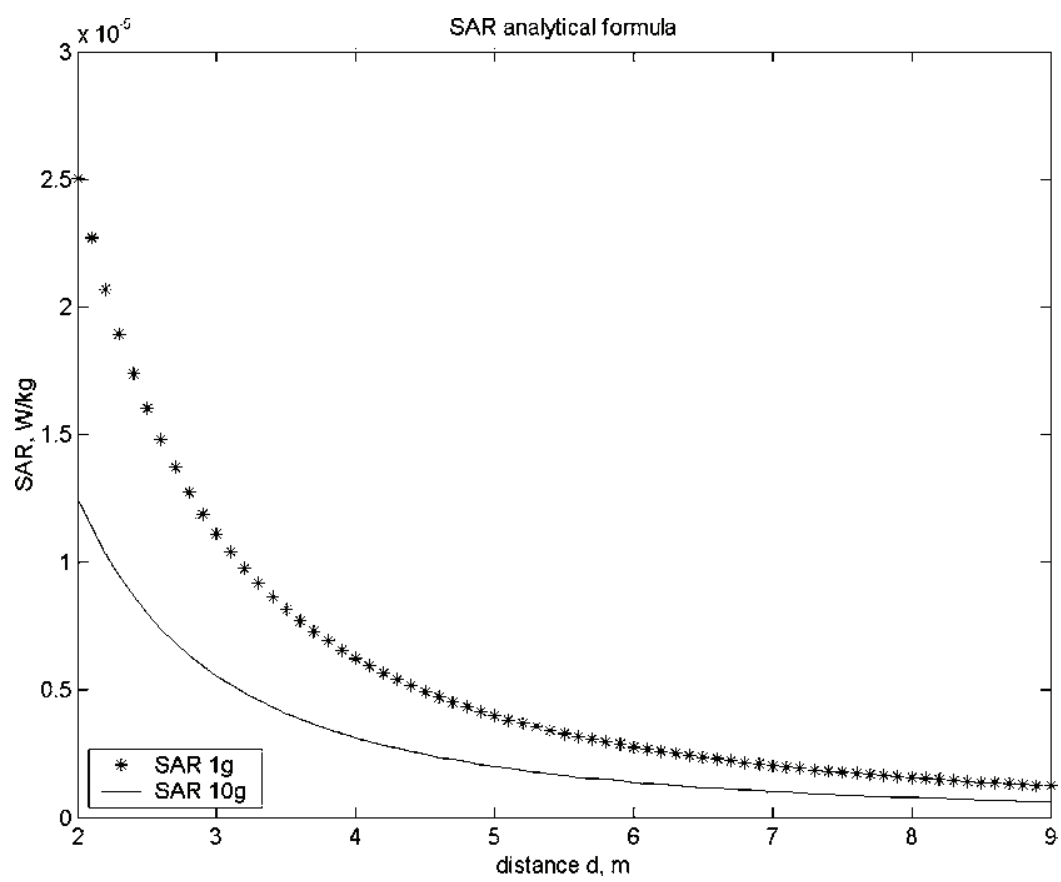

Figure 4: SAR averaged over $1 \mathrm{~g}$ and $10 \mathrm{~g}$ cubes for source distances from 2 to $9 \mathrm{~m}$ for the example WLAN case at $2.432 \mathrm{GHz}$.

The results obtained using the approximation formula eqn (7) for $S A R_{\Delta x}$ averaged over cubes of $1 \mathrm{~g}$ and $10 \mathrm{~g}$ are shown in Fig. 4.

According to [23], the peak $1 \mathrm{~g}$ and $10 \mathrm{~g}$ SARs are calculated from the incident power density, using field strengths obtained from WLAN measurements and beam-tracing simulations, as follows:

$$
\begin{gathered}
S A R_{1 \mathrm{~g}}=\left(1-\mathrm{e}^{-2 / \delta}\right)\left(1-\Gamma \Gamma^{*}\right) S_{\mathrm{inc}} \\
S A R_{10 \mathrm{~g}}=\frac{4.64}{10}\left(1-\mathrm{e}^{-4.309 / \delta}\right)\left(1-\Gamma \Gamma^{*}\right) S_{\mathrm{inc}} .
\end{gathered}
$$

Figure 5 shows the results of the application of eqns (9a) and (9b) to the results obtained by WLAN measurements and beam-tracing simulations.

Figures 4 and 5 indicate the following interesting observation for comparison between the two different methods (analytical from the known source data and calculation of SAR as a dosimetric quantity directly from the exposimetry measurements). Figure 4 shows that the highest SAR averaged over a $1 \mathrm{~g}$ cube $(25 \mu \mathrm{W} / \mathrm{kg})$ occurs at the closest distance of $2 \mathrm{~m}$. The highest SAR value averaged over a $10 \mathrm{~g}$ cube, $12.5 \mu \mathrm{W} / \mathrm{kg}$, occurs at the same distance from the transmitter $(2 \mathrm{~m})$. In the second case, i.e. of results from measurements and beam-tracing simulations, the highest value of SAR averaged over a $1 \mathrm{~g}$ cube appears at the closest distance of $2 \mathrm{~m}$ and is $54 \mu \mathrm{W} / \mathrm{kg}$. The highest SAR value averaged over a $10 \mathrm{~g}$ cube appears at the same distance from the transmitter $(2 \mathrm{~m})$ and it is $25 \mu \mathrm{W} / \mathrm{kg}$. It is important to notice that the analytical method underestimates the values obtained by measurements or 


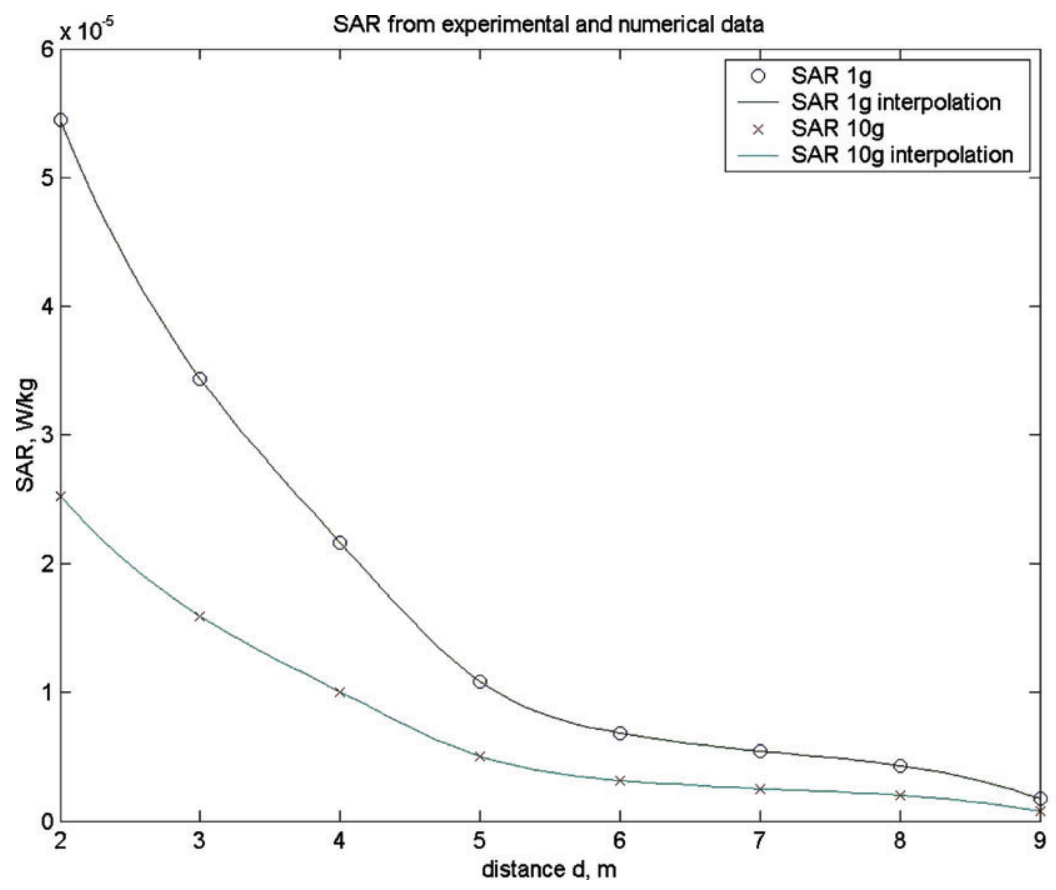

Figure 5: Results of application of eqns (9a) and (9b), SAR averaged over $1 \mathrm{~g}$ and over $10 \mathrm{~g}$ at distances from 2 to $9 \mathrm{~m}$ for the considered WLAN case at $2.432 \mathrm{GHz}$. Results are obtained by WLAN measurements and beam-tracing simulations and by interpolation.

Table 2: ICNIRP basic restrictions of SAR for the frequency range $10 \mathrm{MHz}-10 \mathrm{GHz}$.

\begin{tabular}{lccc}
\hline Frequency range & $\begin{array}{c}\text { Whole-body } \\
\text { average SAR } \\
\text { (W/kg) }\end{array}$ & $\begin{array}{c}\text { Localized SAR } \\
\text { (head and trunk) } \\
\text { (W/kg) }\end{array}$ & $\begin{array}{c}\text { Localized SAR } \\
\text { (limbs) } \\
\text { (W/kg) }\end{array}$ \\
\hline $10 \mathrm{MHz}-10 \mathrm{GHz}$ & 0.08 & 2 & 4 \\
\hline
\end{tabular}

beam-tracing simulations by a factor of approximately 2 . The reason is a non-anechoic environment with reflections from the surface. Still, the differences in obtained values by the three methods (analytical calculation, measurement results, and beam-tracing simulations) are minimal, since they are all characterized by the same order of magnitude. This provides a high confidence in all the results.

To perform a complete dosimetry, the calculated SAR values have been compared to values of the ICNIRP Guidelines [24] for general public exposure. The ICNIRP defines a $10 \mathrm{~g}$ average SAR for the whole body and for the localized parts of the body, i.e. head and trunk and limbs. The values for the frequency range from $10 \mathrm{MHz}$ to $10 \mathrm{GHz}$ are listed in Table 2 .

The comparison is based on the 'worst case' calculation, i.e. the value taken is the maximum of the calculated highest SAR values, which is $54 \mu \mathrm{W} / \mathrm{kg}$. This value is five orders of magnitude lower than the ICNIRP localized SAR value for head and trunk of $2 \mathrm{~W} / \mathrm{kg}$. 


\section{CONCLUSIONS}

In this paper, a beam-tracing software developed for numerical calculations of signal strength in the entire space around a transmitter has been described. Because of the more efficient algorithm compared to classical ray-tracing and, therefore, faster calculation times, the software is ideal for calculations of signal strength in large indoor areas.

In the example presented, the software was applied to a calculation of 160,801 map points, which was performed in $513 \mathrm{~s}$ on a Pentium IV computer, operating at $1.8 \mathrm{GHz}$. This gives an average calculation speed of more than 300 points per second.

The comparison between calculations and measurements gives very encouraging results. The highest standard deviation between measured points and neighboring calculated points was $4.2 \mathrm{~dB}$. Thus, the combination of a numerical model and a measurement method has been applied not only for verification of both the results but also for 'tuning' numerical parameters.

In the next step, the focus has been to determine whether the obtained exposimetry results can be compared to analytical dosimetry results. According to the analytical calculations performed using the theory of EM waves, the highest value appears on the points closest to the transmitter. The dosimetric results obtained after performing the actual exposimetry measurements and simulations show the same behavior, i.e. the steep descent of SAR from the highest value, closest to the transmitter $(2 \mathrm{~m})$. It is important to notice that the analytical method underestimates the values obtained by measurements or beam-tracing simulations by a factor of approximately 2 . The reason is a non-anechoic environment with reflections from the surface. Still, the differences in obtained values by the three methods (analytical calculation, measurement results, and beam-tracing simulations) are minimal, since they are all characterized by the same order of magnitude. This lends credibility to all the results.

The maximum SAR value is $54 \mu \mathrm{W} / \mathrm{kg}$, which is five orders of magnitude lower in comparison with the SAR value in ICNIRP Guidelines $(2 \mathrm{~W} / \mathrm{kg}$ ). Still, it has to be kept in mind that these values of SAR are at distance of $2 \mathrm{~m}$ from the transmitter. The SAR value in the near-field zone or in the area of direct contact of the antenna and the human body deserves special attention and the development of different analytical formulas and measurement techniques. The same is valid for the human exposure to multiple APs or a combination of multiple APs and other RF sources.

\section{REFERENCES}

[1] Radiofrequency Radiation Dosimetry Handbook, 4th edn, USAF School of Aerospace Medicine, Aerospace Medical Division (AFSC), Brooks Air Force Base, October 1986.

[2] Šimunić, D., Dosimetry and exposimetry of pulsed fields. Radio Frequency Radiation Dosimetry, eds. B.J. Klauenberg \& D. Miklavcic, Kluwer Academic Publishers: The Netherlands, pp. $53-62,2000$.

[3] Dersch, U. \& Zollinger, E., Propagation mechanism in microcell and indoor environments. IEEE Transactions on Vehicular Technology, 43(4), pp. 1058-1066, 1994.

[4] Seidel, S.Y. \& Rappaport, T.S., Site-specific propagation prediction for wireless in-building personal communication system design. IEEE Transactions on Vehicular Technology, 43(4), pp. 879-891, 1994.

[5] Report from CEPT to the European Commission in response to the Mandate to: Short Range Devices Radio Spectrum Harmonisation. Report approved on 12 November 2004 by the CEPT ECC.

[6] Directive 1999/5/EC of the European Parliament and of the Council of 9 March 1999 on radio equipment and telecommunications terminal equipment and the mutual recognition of their conformity. Official Journal of the European Communities, L91/10, 1999. 
[7] 2000/299/EC, Commission Decision of 6 April 2000 establishing the initial classification of radio equipment and telecommunications terminal equipment and associated identifiers. Official Journal of the European Communities, L97/13, 2000.

[8] Establishing a Dialogue on Risks from Electromagnetic Fields, World Health Organization, Radiation and Environmental Health, Department of Protection of the Human Environment, Geneva, Switzerland, 2002.

[9] Zrno, D. \& Šimunić, D., Matrix based ray-tracing model for indoor propagation. ICECOM 2003, Dubrovnik, pp. 221-224, 2003.

[10] Larcheveque, E., Dale, C., Wong, M.-F. \& Wiart, J., Analysis of electric field averaging for in situ radiofrequency exposure assessment. IEEE Transactions on Vehicular Technology, 54(4), pp. 1245-1250, 2005.

[11] Bernardi, P., Cavagnaro, M., Pisa, S. \& Piuzzi, E., Human exposure to radio base-station antennas in urban environment. IEEE Trans MTT, 48(11), pp. 1996-2002, 2000.

[12] Doble, J., Introduction to Radio Propagation for Fixed and Mobile Communications, Artech House: Boston and London, 1996.

[13] Hashemi, H., The indoor radio propagation channel. Proc. IEEE, 81(7), pp. 943-968, 1993.

[14] Jackson, J.D., Classical Electrodynamics, John Wiley \& Sons: New York, 1999.

[15] Laurenson, D.I., McLaughin, S. \& Sheikh, A.U.H., The application of ray tracing and the geometrical theory of diffraction to indoor channel modelling. IEEE Global Telecommunications Conference, pp. 1242-1246, 1993.

[16] Ji, Z., Li, B.-H., Wang, H.-X., Chen, H.-Y. \& Sarkar, T.K., Efficient ray tracing methods for propagation prediction for indoor wireless communications. IEEE AP Magazine, 43(2), pp. 41-49, 2001.

[17] IEEE Std. 802.11b-1999 (R2003), Supplement to IEEE Standard for Information technology Telecommunications and information exchange between systems - Local and metropolitan area networks - Specific requirements - Part 11: Wireless LAN Medium Access Control (MAC) and Physical Layer (PHY) specifications: Higher-Speed Physical Layer Extension in the $2.4 \mathrm{GHz}$ Band, IEEE-SA Standards Board, 2003.

[18] Kuster, N. \& Balzano, Q., Energy absorption mechanism by biological bodies in the near field of dipole antennas above $300 \mathrm{MHz}$. IEEE Transactions on Vehicular Technology, 41(1), pp. $17-23,1992$.

[19] Federal Communications Commission, 1997, http://www.fcc.gov/fcc-bin/dielec.sh

[20] Gabriel, C., Compilation of the Dielectric Properties of Body Tissues at RF and Microwave Frequencies, Final Tech. Rep. AL/OE-Tech rep.-1996-0037, Occupational and Environmental Health Directorate, RFR Division, Brooks AFB, TX, USA, 1996.

[21] Kuster, N. \& Balzano, Q., Energy absorption in the near field of MTE. Mobile Communications Safety, eds. N. Kuster, Q. Balzano \& J.C. Lin, Chapman \& Hall: London, 1997.

[22] Bit-Babik, G. \& Faraone, A., Compliance distance of bystanders from mobile antennas at frequencies from $30 \mathrm{MHz}$ to $900 \mathrm{MHz}$. Proc. EMC EUROPE 2002, Sorrento, Italy, pp. 449-452, 2002.

[23] Kang, G. \& Gandhi, O.P., Effect of dielectric properties on the peak 1- and 10-g SAR for $802.11 \mathrm{a} / \mathrm{b} / \mathrm{g}$ frequencies 2.45 and 5.15 to $5.85 \mathrm{GHz}$. IEEE Trans. Electromag. Compat., 46(2), pp. 268-274, 2004.

[24] ICNIRP (International Commission on Non-Ionizing Radiation Protection), Guidelines for limiting exposure to time-varying electric, magnetic, and electromagnetic fields (up to $300 \mathrm{GHz}$ ). Health Phys., 74(40), pp. 494-522, 1998. 